\title{
La presión como factor estresor en el entorno laboral publicitario
}

\author{
Pressure as a stress factor in the \\ advertising work environment
}

\section{Guillermo Andrés Rodríguez Martínez (Colombia)}

Universidad Jorge Tadeo Lozano guillermo.rodriguez@utadeo.edu.co

\section{Resumen \\ La presión laboral es entendida como un potencial factor generador de estrés. La productividad de las personas puede verse afectada dependiendo de la valoración que ellas hagan sobre si una determinada tarea es apremiante o no. Esta valoración está mediada tanto por la autoconfianza del individuo como por el reconocimiento serio y consciente de sus habilidades. El trabajo autotélico, bastión de la teoría del flujo de Mihaly Csikszentmihalyi, implica la ausencia del estrés y el deleite por trabajar, producir y crear. Ese estado ideal (negentropía}

\begin{abstract}
Work pressure is seen as a potential factor that generates stress. The productivity of individuals can be affected depending on whether they think a particular task is urgent or not. This appraisal is mediated by the individual's self-confidence, as well as their serious and conscious recognition of their skills. The autotelic work, bulwark of the theory of flow by Mihaly Csikszentmihalyi, involves absence of stress and delight to work, produce and create. That state of wellbeing (psychic negentropy) is easily affected when stressors such as work overload,
\end{abstract}

FECHA DE RECEPCIÓN: FECHA DE REVISIÓN: APROBACIÓN:
31 de mayo de 2013 2 de agosto de 2013

6 de agosto de 2013
Para Citar este artículo / to CIte this ARTICle Rodríguez, G. (2013). La presión como factor estresor en el entorno laboral publicitario. Poliantea, (IX), 17, pp. 67-86. 
psíquica) fácilmente se ve contrariado cuando en el entorno aparecen factores estresores como la sobrecarga laboral, los plazos cortos para entregar tareas y la amenaza latente de ser despedido del trabajo. Fenómenos psicosociales como la heteronimia instrumental y el descontrol situacional aportan en la configuración del estrés, pudiendo este último derivar en estados psicológicos como la frustración o la ansiedad. Tal y como lo evidenció una investigación realizada por el Programa de Publicidad de la Universidad Jorge Tadeo Lozano, la presión no siempre aporta positivamente en las labores de tipo creativo y muchas veces puede incrementar la experimentación de ansiedad, especialmente cuando los desafíos revisten mayor complejidad. No obstante, en tanto exista autoconfianza y las aptitudes sean acordes al reto laboral, el individuo podrá mejorar su desempeño, permitiendo configurar el estado de flujo propio del autotelismo. Los resultados fueron obtenidos mediante la aplicación de encuestas, diarios de experiencias, sesiones de grupo y entrevistas a profundidad.

Palabras clave: presión, ansiedad, estrés, productividad, autotelismo. short deadlines to carry out assignments, and threat of being fired from work, appear in the work place. Psychosocial phenomena such as instrumental heteronymy and situational decontrol, contribute to stress creation. Thus, stress can derive to psychological states such as frustration or anxiety. Research conducted at the Advertising Program of Jorge Tadeo Lozano University, showed that pressure does not always affect positively creative work. Pressure can often increase anxiety experience, especially when the challenges are more complex. Nevertheless, if there is self-confidence and there are skills that correspond to a particular challenge, an individual can improve their performance, allowing to configure the flow state of autotelism. The results were obtained through surveys, experience journals, group sessions, and in-depth interviews.

Keywords: pressure, anxiety, stress, productivity, autotelism. 


\title{
La presión como factor estresor en el entorno laboral publicitario
}

\section{Pressure as a stress factor in the advertising work environment}

\begin{abstract}
Guillermo Andrés Rodríguez Martínez (Colombia)
Especialista en Gerencia de Mercadeo, por la Universidad Central de Colombia. Magíster en Mercadeo Agroindustrial, por la Universidad Jorge Tadeo Lozano. Actualmente cursa Doctorado en Psicología en la Universidad San Buenaventura, de Medellín. Es autor del libro El consumidor como víctima de sus propios sentidos, escrito en coautoría con Hugo Mastrodoménico Brid.
\end{abstract}

\section{Introducción \\ La valoración subjetiva de las situaciones apremiantes}

El concepto de "presión laboral" ha sido objeto de investigación en el campo de la psicología. Todas las tensiones intrapsíquicas que se pueden derivar de esa presión tienen repercusiones en el desempeño de una persona. Así, mayores o menores estados de tensión habrán de repercutir en la capacidad productiva, hecho que fácilmente puede extrapolarse al campo de la productividad creativa. Referirse al trabajo bajo presión lleva a relacionar dos eventos de tipo cognitivo, denominados por Richard Lazarus "valoración primaria" y "valoración secundaria"; la primera valoración está referida a la interpretación que hace el sujeto de un evento, que culmina con un juicio del cual se establece que dicho evento es amenazador; la segunda guarda relación con el hecho de que el sujeto advierte que no tiene las habilidades ni los recursos para subsanar el suceso amenazador al 
que se ve abocado (Larsen y Buss, 2005, p. 580). ${ }^{1}$ Maslow (1991, p. 32) hace una explícita reseña a la relación existente entre las necesidades del individuo y sus aptitudes:

Aun cuando ciertas necesidades estén satisfechas podemos esperar que, a menudo (si no siempre), se desarrolle un nuevo descontento y una nueva inquietud, a menos que el individuo esté haciendo aquello para lo que él individualmente está capacitado. En última instancia, los músicos deben hacer música, los artistas deben pintar, los poetas deben escribir, si tienen que estar en paz consigo mismos. Lo que los humanos pueden ser, es lo que deben ser.

Frente a la interrelación existente entre aptitudes y desafío, Mihaly Csikszentmihalyi (2008) estableció una teoría ${ }^{2}$ con la que explica que los estados de plenitud psíquica asociados al placer y la felicidad (negentropía psíquica) se producen cuando los niveles de de-

1 Varios autores han definido la presión en función de los elementos del ambiente lo entorno) que inciden en las necesidades de los individuos. Inclusive, distinguen presión alfa de presión beta, dependiendo de si se alude al entorno real o al entorno percibido, respectivamente (Larsen y Buss, 2005, p. 3421.

2 Se está haciendo alusión a la teoría del flujo. safío o reto están en el máximo nivel de expectativa subjetiva. Esa expectativa se produce como consecuencia de la conciencia que la persona tiene con respecto a cuáles son sus potencialidades, sus habilidades, sus capacidades. En palabras de Rodríguez Hernández (2006, pp. 70-71), estas habilidades "constituyen un racimo de respuestas potenciales aprendidas o innatas que, en formato de esquemas conceptuales o procedimentales, garantizan la eficaz adecuación de la conducta a las demandas de la tarea".

Así, cuando a la persona se le ofrece un reto muy desafiante, pero que al tiempo es coherente con sus aptitudes y habilidades, experimenta lo que Csikszentmihalyi denomina estado de flujo, experiencia psíquica que es equivalente a la plenitud emocional, a un estado de felicidad que guarda estrecha relación con los desempeños laborales de tipo autotélico. ${ }^{3}$ En efecto, justo como lo señala Maslow (1991, p. 38), existen personas cuya naturaleza creativa

3 Tal y como lo reseña Rodríguez (201 1b, p. 811, el flujo se define como "el estado en el cual las personas se hallan tan involucradas en la actividad que están haciendo que no parece importarles nada más". La labor que están desempeñando es en sí misma la recompensa, hecho que da cuenta del trabajo de tipo autotélico. 
los lleva a experimentar la creatividad como propósito esencial, por encima de cualquier otro determinante. No obstante, para algunos de ellos el ejercicio de la creatividad no supone la búsqueda de la autorrealización, sino un acto compensatorio de la insatisfacción de necesidades básicas.

Como puede advertirse, la valoración de la demanda laboral está revestida del componente subjetivo, hecho que supone que personas de talante más optimista asuman que para un nivel de habilidades determinado e invariable $\mathrm{X}$ es posible atender cierta necesidad, mientras que uno de corte pesimista verá comprometida (e imposible, en un momento dado) la resolución de la tarea, para el mismo nivel de habilidades X. Acá estaría implicado el concepto de la autoeficacia, ${ }^{4}$ que comprobadamente mejora el desempeño en actividades mentales, físicas, académicas, etc. (Baron

4 Este concepto está referido a la convicción que tiene una persona de poder resolver contingencias, situaciones de apremio, eventos amenazadores de la estabilidad psíquica, etc. (Larsen y Buss, 2005, p. 582). El término se le debe a Albert Bandura, psicólogo de origen canadiense, inscrito en la escuela sociocognitivista. y Byrne, 1998, p. 202). ${ }^{5}$ En efecto, se ha demostrado que el desempeño en actividades físicas, mentales, académicas, etc., puede mejorar conforme exista una adecuada autoeficacia (Baron y Byrne, 1998, p. 202). ${ }^{6}$

En ese orden de ideas, las valoraciones subjetivas alimentan la incidencia que puede llegar a ejercer la presión sobre el desempeño laboral. Desde una perspectiva estrictamente psicológica, se arguye que son también los prejuicios los que derivan no solo en el establecimiento de estados de tensión y estrés, sino también en los llamados bloqueos creativos. ${ }^{7}$

5 Estos autores reseñan que, por ejemplo, las personas con una elevada autoeficacia atlética logran prolongar por más tiempo actividades físicas intensas, como consecuencia de la estimulación en la producción de opióceos endógenos por parte del cuerpo.

6 También, fundamentándose en investigaciones, sugieren que, en desempeños de tipo laboral que requieren esfuerzos cognitivos importantes, la autoeficacia conduce a una mayor productividad.

7 El estudio de los entornos creativos y su incidencia en los bloqueos creativos va más allá de la revisión de las tensiones ocasionadas por la interdependencia entre el sujeto y las demandas laborales. De hecho, tal y como lo señalan Pol, Valera y Vidal (1999, p. 240), existen muchos procesos que median en la interacción entre ambiente y sujeto, como todas las circunstancias de tipo físico que en un momento dado pueden convertirse en factores estresores, tales como la calidad del aire, el ruido, las emisiones gaseosas, etcétera. 
Mastrodoménico (2011) define los prejuicios como las opiniones que las personas se hacen sobre un determinado objeto social, cultural o físico sin poseer una suficiente información que permita hacer una valoración justa y acertada. Las posturas optimistas y pesimistas a las que se hace referencia muchas veces están referidas a un prejuicio configurado, que predispone a las personas a vivir con mayor o menor tensión cierta situación laboral apremiante. Sin duda, el prejuicio optimista (que da cuenta de la tendencia de las personas a ignorar o minimizar los riesgos que implican las diversas situaciones de la vida) ayuda al sujeto a admitir desafíos de importante consideración. ${ }^{8}$

8 No obstante, como se insinuó, el prejuicio es relativo a cada persona e incide en la definición de las categorías persona optimista y persona pesimista; esta última sobreestima los riesgos. Una estrategia que encauza a las personas en la búsqueda de la resolución de sus desafíos es la revaloración positiva, consistente en poner en un primer plano de la atención todos los aspectos favorables de la situación valorada primeramente como apremiante. Este mecanismo fue propuesto por las psicólogas Susan Folkman y Judith Moskowitz, quienes también sugirieron un afrontamiento enfocado en el problema y una tercera estrategia llamada creación de sucesos positivos (Larsen y Buss, 2005, pp. 583-585).

\section{La generación de estrés}

El estrés ${ }^{9}$ supone un estado de tensión ocasionado por un desequilibrio o alteración de orden psíquico proveniente del juicio que se hace de una determinada situación, donde el veredicto se circunscribe a establecer si esta es amenazante o contraria a los intereses y expectativas de una persona. Larsen y Buss (2005, p. 574) caracterizan los factores productores de estrés como extremos o sobrecargados, aduciendo que generalmente producen tendencias opuestas en la persona (pugna constante de motivos y frenos), convirtiéndose en incontrolables con relativa facilidad. Fun-

9 La psicología ha tipificado cuatro variedades de estrés: a) el estrés agudo, producido por el advenimiento repentino de tareas, demandas, asuntos para resolver, etc.; b) el estrés agudo episódico, asumido como la presentación frecuente o repetida de estrés agudo; c) el estrés traumático, referido a la manifestación masiva e intensificada del estrés agudo, y d) el estrés crónico, que implica la presencia de un estrés que sencillamente no termina, que se presenta en la persona de forma ilimitada (Larsen y Buss, 2005, pp. 579-580). Feldman (1999, p. 326) define el estrés como una consecuencia derivada de sucesos amenazantes que generan conflicto a una persona. Una investigación realizada por Wegner, Broome y Blumberg (1997) demostró que intentos deliberados por producir relajación en momentos de estrés fácilmente conducen al advenimiento de una mayor exaltación (Kerlinger y Lee, 2002, pp. 359-3611. 
damentalmente, el término estrés ingresa a la terminología popular y psicológica por el papel que desempeña en la etiología psicosomática. El estrés puede configurarse por la interacción entre las características de la persona y las demandas del medio.

Algunas características que con frecuencia presentan los eventos estresores, son: cambio o novedad en la situación estimular; falta de información e impredictibilidad de la situación; incertidumbre; ambigüedad de la situación y sobrecarga de información; falta de habilidades o conductas para afrontar la situación que se presenta; y alteración de las condiciones biológicas del organismo (Consuegra, 2011, pp.106-107).

Se produce estrés cuando las demandas pedidas a determinada persona producen tensión psíquica como consecuencia de una valoración hecha por el sujeto, donde este establece que existe una relativa dificultad (y hasta imposibilidad) de poder llevar a cabo una tarea, lo que sobreviene a un estado de frustración ${ }^{10}$. Ese estado emocional

10 Los elevados niveles de frustración pueden conducir a actos de agresión, aunque casi siempre de manera indirecta, antes de un proceso de activación, que puede tener puede adquirir relevancia y suponer el advenimiento de los bloqueos creativos, como consecuencia de una combinación entre tensión y ansiedad. ${ }^{11}$ De alguna manera, los denominados "factores estresores" (como la sobrecarga laboral) atentan contra las necesidades de seguridad, justo como las definió Abraham Maslow, pues estas van referidas a la estabilidad, la dependencia, la protección, la ausencia de miedo, la necesidad de estructura, de orden, de ley y de límites (Maslow, 1991, pp. 25-26). Desde esa perspectiva, las actividades que se correspondan con un encauzamiento hacia el equilibrio psíquico y la estabilidad emocional tendrán la susceptibilidad de redundar en la

antecedentes estresores, tales como las condiciones ambientales adversas y los ataques personales, bien sean físicos o psíquicos (Morales y Arias, 1999, p. 130).

11 En la teoría del flujo de Mihaly Csikszentmihalyi aparecen tipificados ocho estados de experiencia, los cuales dependen de la diferencia entre las aptitudes del individuo y el nivel de reto o desafío laboral. Entre esos estados figura, justamente, la ansiedad, que, desde una perspectiva estrictamente psicoanalítica, ha sido definido como un estado de malestar muy próximo al miedo con un elevado potencial desestabilizador (Rodríguez, 2011 b, p. 84). Dentro de la teoría del flujo, los estados opuestos a la ansiedad son el aburrimiento y el interés. Maslow (1991, p. 63) hace una relación entre estos dos estados de experiencia con la gratificación de necesidades. Atribuye al exceso de gratificación el advenimiento del aburrimiento. 
disminución parcial o total de la experimentación de estrés. Reséñese también que cuando se presenta una variación en las demandas laborales, de manera tal que la organización cognitiva que tenía la persona se ve modificada, se presenta la manifestación de una potencial tensión, que puede ser vista como una alteración del entorno, lo que supone que este no simplemente está definido por las condiciones exógenas al sujeto. Cítese a Pol, Valera y Vidal (1999, p. 243):

Al situarnos ante determinado entorno se activan un conjunto de mecanismos fisiológicos y psicológicos que permiten captarlo y obtener información de él. Las sensaciones recibidas son integradas en unidades de contenido y significado que nos permiten reconocer, comparar o explorar el entorno, experimentar sensaciones o emociones y actuar en consecuencia integrando las motivaciones e intereses personales, las características ambientales y el contenido social que se deriva del propio contexto, en definitiva, tener una experiencia ambiental.

Autores como Dennis Coon (2001, p. 303) proponen una serie de técnicas para reducir el estrés y mejorar las habilidades de afrontamiento. Entre ellas están el manejo de las reacciones corporales (mediante la búsqueda deliberada del relajamiento muscular), la meditación (que puede promoverse por el disfrute de pasatiempos), la relajación progresiva (consistente en tensionar zonas del cuerpo y luego, a discreción, relajarlas sistemáticamente para tener conciencia del momento de soltura y distensión) e ingeniería guiada (más ceñida a un acto imaginativo, en el que la persona produce visualizaciones en su plano intrapsíquico que le produzcan tranquilidad y placer).

Desde el punto de vista psicosocial, un componente decisivo que se relaciona con el estrés causado por determinada situación de apremio, es la autoestima. Esta noción, esencialmente definida como la autoevaluación que el individuo hace de sí mismo por mediación de la comparación entre el yo ideal y el yo real, guarda estrecha relación con la autoeficacia, concepto referido con anterioridad. Si la autoestima es más bien positiva, se abona en el terreno psíquico la posibilidad de experimentar mayor seguridad a la hora de enfrentar momentos estresores, hecho que respalda Maslow (1991, p. 131): "La satisfacción de la necesidad de autoestima conduce a sentimientos de autoconfianza, valía, fuerza, capacidad y suficiencia, de ser útil y necesario 
en el mundo". ${ }^{12} \mathrm{La}$ autoestima puede ser tomada como uno de los aspectos que configuran la autoimagen (o self-image), ${ }^{13}$ concepto que advierte todo el conjunto de representaciones mentales que tiene un individuo sobre sí mismo. Desde el punto de vista de un marco contextual laboral que suponga la permanente generación de ideas creativas, la autoimagen que entraría a primar sería el self-image, referido al ser creativo, esto es, el concepto que una persona tiene sobre sí misma en términos de sus actitudes y aptitudes para la creatividad. Adler, importante psicoanalista que tomó distancia de la postura pansexua-

12 Cuando a una persona se le hace una exigencia laboral, necesariamente está en una interdependencia con quien promulga la exigencia. Es alli cuando entra en juego la heteronimia instrumental, que implica el conjunto de relaciones interpersonales donde existe una relación de sumisióndominancia, por la presencia de un individuo que ejerce autoridad sobre otro. En esta heteronimia entran en juego el respeto y la prudencia por parte del que está en un grado inferior en la relación de poder, y tolerancia, imposición (y a veces abuso) por parte de quien ejerce autoridad (Heredia y Huerta, 2002, p. 171). El término heteronimia está referido a la recepción que el individuo hace de reglas, normas, órdenes, imposiciones, etc., venidas de su entorno.

13 Esta noción hace parte de un concepto más global, el self. Para Consuegra (201 1, p. 241), el self tiene siete funciones: a) sensación del cuerpo, b) identidad propia, c) autoestima, d) extensión de uno mismo, e) autoimagen, fl adaptación racional y gl esfuerzo propio. lista para enmarcar los rasgos de la personalidad en función de un afán por lograr la superioridad, hizo referencia explícita al self-creativo, componente esencial de las personas que quieren sobreponerse a los obstáculos de cara a una plena autorrealización, a partir de una reinvención de sí mismas.

El hombre es más que un producto de su ambiente, más que un animal totalmente predispuesto $\mathrm{y}$ confinado por su pasado instintivo hereditario. El hombre es un interpretador de la vida. Con esto quiere decir Adler que el hombre crea una estructura del self (de sí mismo) a partir de su pasado hereditario, interpreta las impresiones que recibe durante el transcurso de su vida, busca nuevas experiencias para realizar sus deseos de superioridad y reúne todo esto para crear un self que es diferente de cualquier otro self y que describe su propio y particular estilo de vida. El self creativo es un paso adicional más allá del estilo de vida. El último es reactivo y mecánico. Sin embargo, el self creativo es algo más que esto; es original, inventivo y crea algo que nunca antes existió: una nueva personalidad; crea un self (Cueli y Reidl, 1982, p. 74). 


\section{Estrés, presión y desempeño creativo}

El Programa de Publicidad de la Universidad Jorge Tadeo Lozano llevó a cabo una investigación ${ }^{14}$ con la que se indagó en más de 350 profesionales del medio publicitario en Colombia (un ámbito positivamente activo, ${ }^{15}$ según las consideraciones teóricas de Mihaly Csikszentmihalyi), sobre si mayores desafíos creativos y mayores presiones laborales inciden positiva o negativamente en la

14 Esta investigación tuvo por título "El flujo creativo de los profesionales del medio publicitario según el nivel de desafío de su trabajo". Se hizo entre 2010 y 2011. La pregunta central de investigación fue: ¿Qué incidencia tiene el incremento del desafío o reto creativo sobre la generación del estado de flujo y de los demás estados de experiencia de los profesionales de la publicidad, tomando en consideración su trayectoria en el medio publicitario? La investigación fue de tipo exploratorio e implicó análisis tanto cuantitativos como cualitativos. Apeló a un muestreo por conveniencia para la aplicación de los instrumentos de investigación, a saber: encuestas, diarios de experiencias, sesiones de grupo y entrevistas.

15 Se entiende por ámbito positivamente activo aquel que es proclive a estimular la creatividad. Las labores publicitarias, por requerir de esfuerzos de tipo creativo en lo relativo a la ideación de estrategias y mensajes impactantes, es un ámbito de este tipo (Rodríguez, 2011 la, p. 12). Su opuesto es el ámbito reactivo, que no exige ni reclama de creatividad (Csikszentmihalyi, 1996, p. 62-631. productividad laboral. La muestra ${ }^{16}$ estuvo configurada por profesionales de diferentes áreas, entre las que se incluyeron creativos, redactores publicitarios, directores creativos, creativos gráficos, planners, realizadores, productores gráficos, productores cinemático-visuales, creativos digitales, ilustradores, estrategas de medios, ordenadores de medios y ejecutivos de cuenta (de todos ellos se estableció su nivel de

16 Para efectos de determinar la muestra, los investigadores determinaron que la aplicación de los instrumentos debía realizarse sobre sujetos que trabajaran en el medio publicitario, independientemente de si eran o no publicistas, a sabiendas de que dentro de la aplicación del muestreo figurarían más publicistas que otro tipo de profesionales. Igualmente, se determinó que de todos estos profesionales aplicarían como sujetos solamente aquellos cuyo trabajo se circunscribiera a labores creativas y de planificación, bien fuera en departamentos creativos, gráficos, de producción o de cuentas. Esta muestra se estableció por conveniencia: conforme se diera la autorización por parte de una u otra agencia (o empresa) para aplicar los instrumentos de investigación, el equipo investigador procedía a hacer la respectiva recolección de información; metodológicamente se estableció como prioridad superar las 350 encuestas y poder medir experiencias laborales dentro del medio publicitario en un número superior a 500 . Estas dos metas se cumplieron de manera satisfactoria. Las entrevistas a profundidad (22) y las sesiones de grupo (2) se realizaron como un complemento a la información que se pudo obtener con la aplicación de las encuestas y los diarios de experiencias. La investigación se llevó a cabo en Bogotá. 
experiencia en tiempo dentro del medio).

Mediante la aplicación de varios instrumentos (encuestas, diarios de experiencias, sesiones de grupo y entrevistas a profundidad) se logró establecer que para $65 \%$ de los investigados la presión no repercute positivamente en el desempeño creativo. La respuesta de orden cualitativo que ofreció la mayoría está resumida en la expresión: "la presión no contribuye con la incubación de ideas. Se requiere tiempo para procesar, para concluir, para comparar, etc.", respuesta que fue dada por $76 \%$ de quienes dijeron que la presión no estimula la productividad creativa. Para quienes mencionaron que la presión sí estimula la creatividad
(35\% del total de la muestra), el argumento que más destacó fue el de que la presión genera más exigencia y la mente se obliga a trabajar más, a enfocarse y concentrarse. Por supuesto, cada razón es valedera, y cada cual, desde su experiencia y perspectiva, estima en uno $u$ otro sentido el trabajo a presión. La investigación pudo revelar también que para los profesionales del medio publicitario bogotano el factor estresor que genera más presión es el corto tiempo para entregar el trabajo, seguido del miedo a la cancelación del contrato (estos datos serán refrendados más adelante). En la figura 1 se aprecia el comparativo de respuestas dadas sobre el efecto de la presión en la productividad creativa.

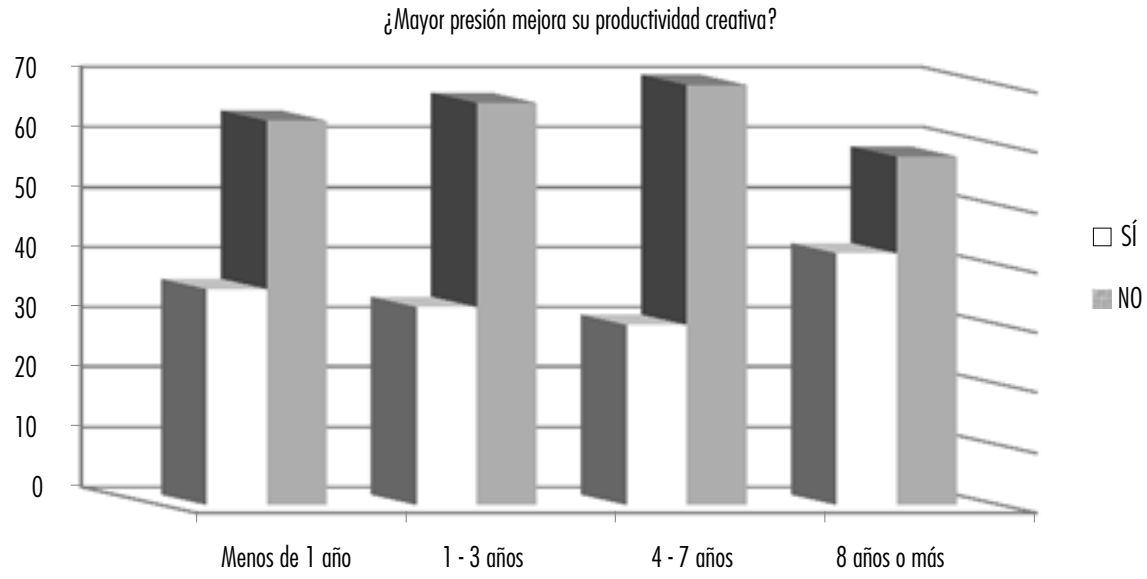

Figura 1. Predominancia de las respuestas negativas sobre las afirmativas, ante la pregunta de si mayor presión mejora la productividad creativa. 
- Nota: se discriminan los resultados tomando en consideración los años de trayectoria profesionaldelos sujetos deinvestigación.

En la edición 13 de la revista Poliantea (julio-diciembre de 2011), en el artículo "La experimentación de la negentropía psíquica asociada al reto creativo" (Rodríguez, 2011b, pp. 89-90) ya se había hecho referencia a algunos resultados obtenidos con la investigación acá reseñada, en relación con la interdependencia de la variable "presión" y la variable "desempeño creativo". Allí se mencionó que, dado que no era posible considerar como equivalentes a los grupos muestrales (grupo $\mathrm{A}=$ menos de un año de trayectoria profesional, grupo $\mathrm{B}=$ entre uno y tres años de trayectoria, grupo $\mathrm{C}=$ entre cuatro y siete años, y Grupo $\mathrm{D}=$ trayectoria de ocho años o más), en razón a que el número de sujetos fue diferente para cada uno de ellos, el procesamiento estadístico no paramétrico utilizado fue el de la prueba de las medianas (mediana combinada). ${ }^{17}$

17 La mediana combinada es la mediana para todos los puntajes de ambos grupos (Siegel, 1970, p. 138). Para establecer la probabilidad de la diferencia, el análisis estadístico se complementa con la prueba chi cuadrado de una cola. Cuando el nivel
Al aplicar el estadístico y establecer el nivel de probabilidad chi cuadrado (0.035982), la significancia de la diferencia a favor del grupo de personas con más trayectoria en el medio publicitario en Bogotá fue alta, hecho que significó que a mayor experiencia profesional de los sujetos, mayor su propensión a desempeñarse mejor en términos creativos cuando se introduce la variable presión en el entorno laboral (ver figura 2). ${ }^{18}$

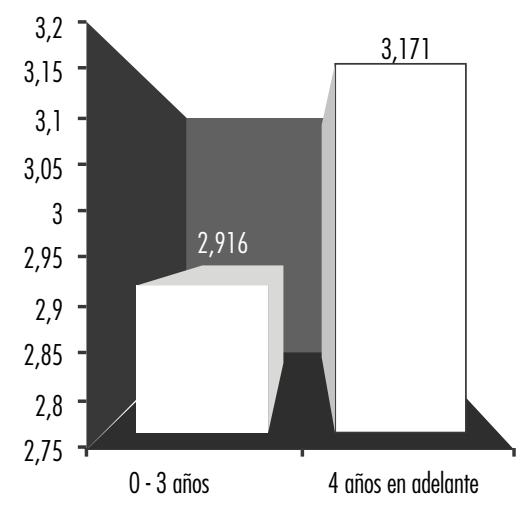

Figura 2. Prueba de las medianas del comparativo de la incidencia del trabajo a presión en la productividad creativa, según la trayectoria o experiencia laboral de los sujetos (cero-tres años frente a cuatro años en adelante).

de probabilidad es menor que 0.05 , se asume que la diferencia de los puntajes cotejados es significativa.

18 Esta figura se soporta en la pregunta nú mero 4 de la encuesta. Los sujetos, en un diferencial semántico, calificaban de 1 a 5 la incidencia del trabajo a presión sobre la productividad creativa, donde 1 equivalía a "disminuye marcadamente la productividad creativa", y 5 a "aumenta marcadamente la productividad creativa". 
- Nota: la diferencia estadística fue significativa. Correspondencia de los puntajes extremos: 1 = Disminuye marcadamente la productividad creativa; 5 = Aumenta marcadamente la productividad creativa. Prueba de las medianas: 4.3979526. Probabilidad de chi cuadrado: 0.035982 .

Puede advertirse, desde los datos presentados con anterioridad, que la presión no se puede estimar de manera definitiva como un fenómeno que incida de manera negativa en los procesos creativos. De hecho, así como se obtienen respuestas que apoyan el trabajo a presión como estímulo de la creatividad, también se encuentran estudios que, si bien muestran la relatividad del asunto, evidencian su efecto negativo. Cítese un estudio realizado en Harvard: Teresa Amabile, Constance N. Hadley y Steven J. Kramer escribieron un artículo apoyándose en la investigación que se hizo para resolver el interrogante de si el trabajo bajo presión favorece o no la creatividad. El artículo, titulado "Creatividad bajo presión", describe en detalle el estudio, que incluyó datos de 177 miembros (estimados en su ámbito como "muy creativos") de 22 equipos de proyecto de siete empresas estadounidenses en tres sectores (químico, alta tecnología y productos de consumo). Los sujetos, cuando estaban ante mucha presión, se sentían más implicados con su trabajo y tenían una sensación de desafío que incrementaba su energía, esto es, su disposición a trabajar con alto grado de motivación. No obstante, muchos de ellos experimentaron una gran frustración conforme aumentaba la presión de tiempo, y la reducción del pensamiento creativo era aún más acentuada cuando esa presión estaba en su paroxismo. Según el estudio, en las jornadas laborales de máxima presión las personas fueron $45 \%$ menos efectivas -creativamente hablando- que en cualquiera de los escenarios estimados como de baja presión. Pero resultó también significativo que algunos sujetos lograron producir ideas auténticamente creativas habiendo tenido muy poco tiempo para su invención. Ello se dio (según el estudio) como consecuencia del cumplimiento de las siguientes condiciones: primero, que pudieron experimentar un estado de concentración; segundo, que la presión logró ser asumida como una urgencia 
significativa; $y$, tercero, que el corto plazo de entrega no tuvo injerencia en procesos creativos que reclamaran elevados niveles de aprendizaje, generación de diversas ideas para posterior tamizado y necesidad de experimentación (Amabile, Hadley y Kramer, 2004, pp. 49-52).

\section{Factores que generan menos y más presión}

Para las personas que encuentran en la presión un factor que contribuye con la productividad creativa, sobresalen argumentaciones en el sentido de que la mente se obliga a trabajar más, que hay necesidad de concentrarse (enfocarse) en los problemas más agudamente, que hay mayor exigencia, en tanto que los sujetos que valoraron de manera negativa la presencia de la presión sustentaron su respuesta en que los procesos creativos requieren de un tiempo prudencial para su incubación. Así, el factor tiempo afloró desde las respuestas cualitativas como uno de los aspectos que genera mayor presión laboral. ${ }^{19}$

\footnotetext{
19 Al revisar toda la información recaudada, los sujetos hicieron una distinción entre las variables "desafío" y "presión", donde la primera estuvo, fundamentalmente, circunscrita a trabajos de mayor envergadura, de
}

Entre las variables que se cotejaron en la investigación (y que fueron seleccionadas gracias a las respuestas obtenidas en entrevistas piloto realizadas antes de diseñar los instrumentos finales para la recolección de información), sobresale que el miedo a la cancelación en el contrato lidera las respuestas en cuanto al factor estresor que menos presión genera en los sujetos (destáquese que el grupo que en mayor porcentaje manifestó que la cancelación del contrato es el factor que menos presión genera es el de menor experiencia laboral, esto es, de menos de un año). El miedo a regaños o amonestaciones también se destaca como un factor generador de poca presión, junto con la pretensión de quedar bien ante los demás. Desde la tabulación y análisis de los resultados, resulta concluyente el hecho de que, para muchos sujetos, los plazos de entrega cortos son un factor generador de presión. Al revisar la

mayor importancia, de mayor vinculación de aspectos y variables problemáticas; la variable "presión" fue asumida como condiciones apremiantes para la resolución de cualquier tipo de problema (independientemente de su nivel de desafío o reto); como se acotó, la condición mayormente asociada a la presión fue el factor tiempo, desde las respuestas cualitativas obtenidas en las encuestas, entrevistas y focus group. 
escala de factores que menos presión generan, "plazos de entrega cortos" aparece entre los últimos; al cotejar la escala jerárquica en el otro sentido (factores que mayor presión generan), los plazos de entrega cortos se constituyen en el factor más sobresaliente (así lo manifestó $45 \%$ del total de la muestra), seguido de "miedo a la cancelación del contrato" (17\% de respuestas), "exigencia verbal o escrita del jefe directo" (14\%), "quedar bien ante los demás" (11 \%) y "miedo a amonestación o regaño" (4 \%). Pero lo más revelador de la investigación es que conforme aumenta el nivel de trayectoria de los sujetos en el medio publicitario, mayores son las manifestaciones que se hacen en el sentido de aceptar que el tiempo corto es el factor estresor que más presión genera (además de ser el ítem mayormente avalado como generador de presión, es el único aspecto cuya curva va in crescendo en proporción al incremento de la experiencia laboral del individuo) (ver figura 3). En todo caso, al revisar las respuestas obtenidas de toda la muestra se hace notorio que la productividad creativa se ve alentada de manera preponderante por dos eventos: el incremento del desafío y la adjudicación de más plazo para la resolución de los problemas (ver figura 4).

\section{El factor que más presíon genera}

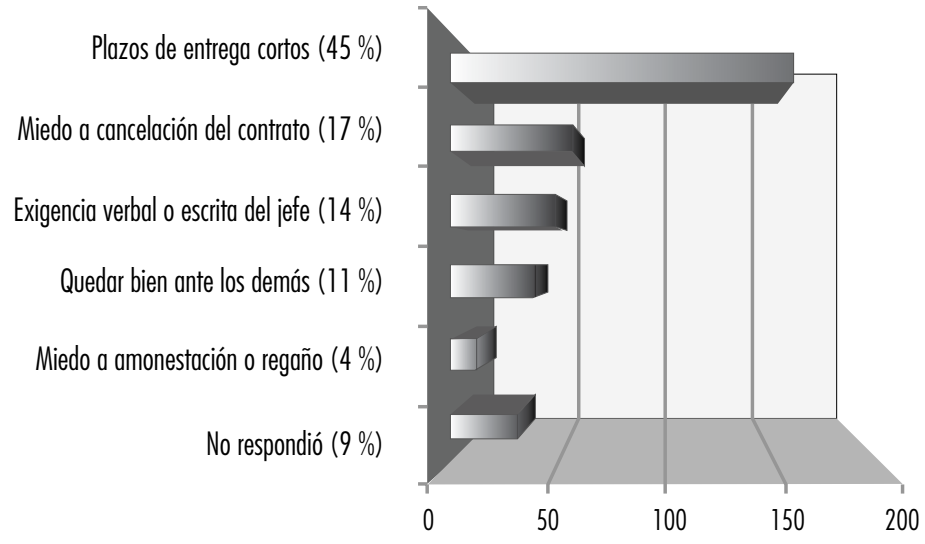

Figura 3. Factores que generan presión 
¿Qué factor mejora la productividad creativa?

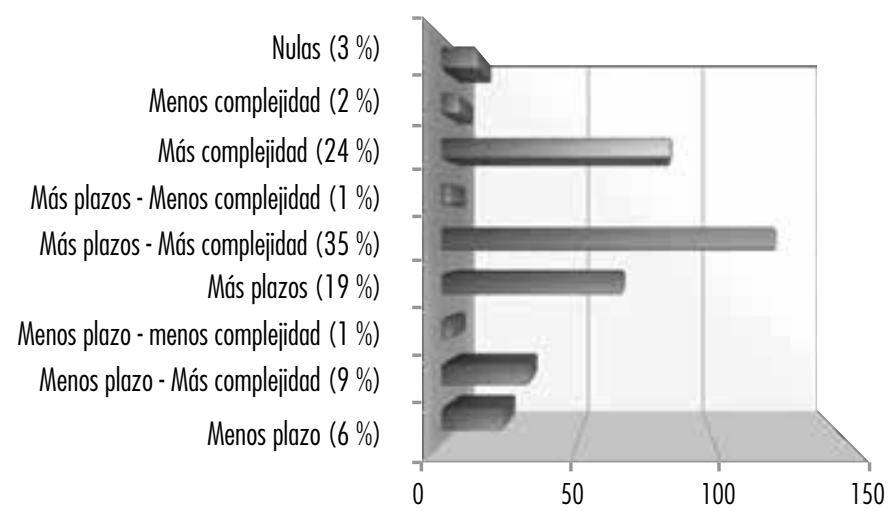

Figura 4. Factores que mejoran la productividad creativa

\section{Experimentación de ansiedad}

La correlación directa entre estrés $\mathrm{y}$ ansiedad es mencionada por Brody y Ehrlichman (2000, pp. 119120) y se asume como un fenómeno que tiende a darse en la mayoría de las personas: aumenta el estrés y se incrementa la ansiedad. No obstante, las personas se diferencian en su tendencia a reaccionar con ansiedad:

Es obvio que distintas personas responden con diferentes niveles de ansiedad a la misma situación (un examen, un viaje en avión, una cita, hablar en público, etc.). Menos obvio es que el concepto de estrés per se no es tan fácil de separar de la personalidad. De hecho, el término estrés puede ser bastante ambiguo. Aunque todos podemos estar de acuerdo en que algunas situaciones son más estresantes, amenazadoras y molestas que otras, muchas de las situaciones cotidianas se viven de un modo estresante porque el individuo está, en realidad, reaccionando con ansiedad y agotamiento.

Con la aplicación de los diarios de experiencia, ${ }^{20}$ la investigación

20 En los diarios de experiencias, los sujetos de la investigación relacionaron, durante dos días de trabajo, seis de sus experiencias laborales, tres en un primer día y otras tres en las siguientes veinticuatro horas. En cada diario el sujeto no solo indicaba el nivel de trayectoria que tenía en el medio publicitario, sino que, además, para cada una de las seis actividades, indicaba el grado de desafío y el correspondiente estado de experiencia vivido, eligiendo entre las alternativas equivalentes a las experiencias sugeridas por la teoría del flujo (desinterés, preocupación, ansiedad, aburrimiento, interés, relajación, control y plenitud), 
pudo verificar que existe directa proporcionalidad entre el incremento del desafío laboral (potencial factor estresor) y la experimentación de la ansiedad (ver figura 5). ${ }^{21}$

- Nota: en este gráfico se toman en consideración todos los datos y valores de todos los sujetos (a partir de la tabulación de los diarios de experiencias). La columna de números a la izquierda expresa el valor en términos de porcentaje, donde $100 \%$ es el total de experiencias registradas para cada desafío.

A manera de cierre, debe convenirse que es importante entender los mecanismos psicológicos que se activan cuando los individuos valoran determinada situación laboral como apremiante. Si bien esa valoración es

pero pudiendo escoger una diferente, en caso de que así le pareciera al sujeto lél denominaría el estado de experiencia con la palabra que mejor le pareciera). Se pudieron documentar más de quinientas actividades laborales con este instrumento.

21 Fue muy notoria la tendencia, a pesar de un pequeño declive en la curva cuando el nivel de desafío fue máximo (nivel 5). El pico máximo del estado de ansiedad se dio cuando el desafío fue valorado como de nivel 4 (en escala de 1 a 5). El estado de flujo, igualmente, tuvo una tendencia importante a crecer, conforme aumentaba el nivel de desafío. relativa a cada persona, lo que resulta imperioso, desde la investigación acá cotejada, es que se pueden consolidar estrategias para que la productividad laboral mejore a partir de revisiones sistemáticas que visibilicen potenciales factores estresores en diferentes ámbitos. Variables como la autoeficacia, la heteronimia instrumental y el autotelismo deben seguir siendo objeto de estudio, para mejorar la vida psíquica de las personas en sus respectivos entornos laborales. Acótese también que, de conformidad con los instrumentos de investigación aplicados, la valoración que hicieron los sujetos de los diferentes factores objeto de estudio se constituye en su propia apreciación, esto es, en una estimación que hicieron desde su experiencia en el entorno laboral publicitario. Así, se puede asumir que, en el marco de la investigación, la presión allí cotejada fue del tipo beta, puesto que escapaba al objeto de estudio revisar las condiciones reales de los escenarios laborales. En esencia, la sustancia de los datos acá encontrados se enmarcó más en el entorno percibido de los sujetos, relacionado con las vivencias de estrés, presión, ansiedad, etc., de cada uno de ellos, según sus experiencias particulares. $\mathrm{Si}$ 


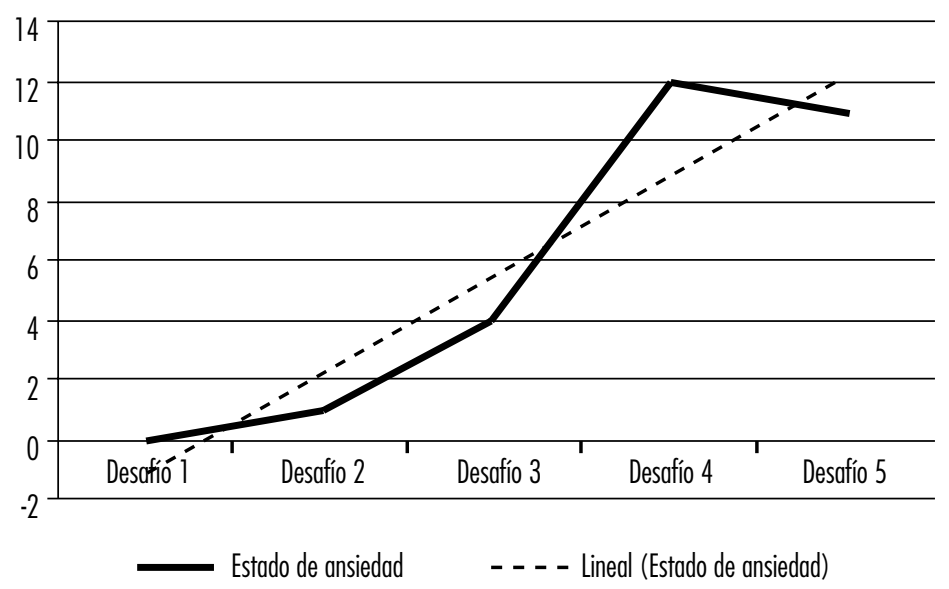

Figura 5. Tendencia a experimentar estado de ansiedad conforme aumenta el nivel de de desafío

bien esta investigación se circunscribió al ámbito laboral creativo de la publicidad en Bogotá, existe el convencimiento (por parte de sus gestores) de que vale la pena tener en cuenta los resultados obtenidos, de cara al fortalecimiento del estudio del estrés asociado a la productividad laboral.

\section{Referencias}

Amabile, T.; Hadley, C. y Kramer, S. (2004). Creatividad bajo presión. Madrid: Ediciones Deusto/Planeta de Agostini Profesional/Formación S. L.

Baron, R. y Byrne, D. (1998). Psicología social. Madrid: Prentice-Hall Iberia.
Beaudot, A. (1980). Creatividad. Madrid: Narcea.

Brody, N. y Ehrlichman, H. (2000). Psicología de la personalidad. Madrid: Prentice-Hall.

Claxton, G. (1997). Cerebro de liebre, mente de tortuga. Barcelona: Urano.

Consuegra, N. (2011). Diccionario de psicología. Bogotá: Ecoe.

Coon, D. (2001). Fundamentos de psicología. México: International Thomson Editores.

Csikszentmihalyi, M. (1996). Creatividad. El fluir de la psicología del descubrimiento y la invención. Barcelona: Paidós Ibérica. 
Csikszentmihalyi, M. (2008). Fluir. Una psicología de la creatividad. Barcelona: Kairós.

Cueli, J. y Reidl, L. (1982). Teorías de la personalidad. México: Editorial Trillas.

Feldman, R. (1999). Psicología. México: McGraw-Hill.

Foster, J. (1996). Cómo generar ideas. Bogotá: Norma.

Garrison, M. y Loredo, O. (2002). Psicología. México: McGraw-Hill.

Ghiselin, B.; Rompel, R. y Taylor, C. (1980). "Check-list" del proceso creativo. En Beaudot, A. La creatividad (pp. 81-92). Madrid: Narcea.

Goleman, D. (2000). El espíritu creativo. Buenos Aires: Javier Vergara Editor.

Graña, N. (2003). La creatividad en la escuela. Montevideo: Aula Editores.

Guilford, J. P. (1980). La creatividad: retrospectiva y prospectiva. En Beaudot, A. La creatividad (pp. 209224). Madrid: Narcea.

Guilford, J. P. (1986). La naturaleza de la inteligencia humana. Barcelona: Paidós.
Heredia, B. y Huerta, J. (2002). Temas básicos de psicología. México: Trillas.

Kerlinger, F. y Lee, H. (2002). Investigación del comportamiento. México: McGraw-Hill.

Larsen, R. J. y Buss, D. M. (2005). Psicología de la personalidad. México: McGraw-Hill.

Marín, R. (1984). La creatividad. Barcelona: Ediciones CEAC.

Maslow, A. H. (1991). Motivación y personalidad. Madrid: Díaz de Santos.

Mastrodoménico, H. (2011). Democracia en el aula de clase. ¿Las conclusiones a las que llegan los grupos de discusión en el aula son tomadas libremente? Berlín: Editorial Académica Española.

Matussek, P. (1977). La creatividad desde una perspectiva psicodinámica. Barcelona: Herder.

Mayer, R. (1983). Pensamiento, resolución de problemas y cognición. Barcelona: Paidós.

Michalko, M. (2000). Cracking creativity. Barcelona: Gestión 2000.

Morales, J. F. y Arias, A. (1999). Agresión. En Morales, J. F. y Huici, 
C. Psicología social (p. 130). Madrid: McGraw-Hill.

Osborn, A. (1953). Applied imagination: principles and procedures of creative thinking. Nueva York: Scribner.

Pal. (1982). Diccionario de psicología. Bilbao: Mensajero.

Pol, E.; Valera, S. y Vidal, T. (1999). Psicología ambiental y procesos psicosociales. En J. F. Morales, \& C. Huici, Psicología social (págs. 235 252). Madrid: Mc Graw-Hill.

Ricarte, J. (1999). Creatividad y comunicación persuasiva. Barcelona: Universidad Autónoma de Barcelona.

Rodríguez Hernández, A. (2006). Dialogando con la teoría componencial de Teresa Amabile. En Torre, S. de la y Violant, V. Comprender y evaluar la creatividad. Un recurso para mejorar la calidad de la enseñanza (pp. 69-83). Málaga: Aljibe.
Rodríguez, A. (2011a). El mapa mental y su incidencia en la creatividad publicitaria (vol. 4, núm 1). Bogotá: Fundación Universidad de Bogotá Jorge Tadeo Lozano.

Rodríguez, A. (2011b). La experimentación de la negentropía psíquica asociada al reto creativo. Una revisión de la teoría del flujo dentro del oficio publicitario en Bogotá. Poliantea, 13, 77-94.

Romo, M. (1997). Psicología de la creatividad. Buenos Aires: Paidós.

Siegel, S. (1970). Diseño experimental no paramétrico aplicado a las ciencias de la conducta. México: Trillas. 